

\title{
Optical transmission properties and electric field distribution of interacting 2D silver nanorod Arrays
}

Pollard, R., Evans, P. R., Kullock, R., Hendren, W. R., Atkinson, R., \& Eng, L. M. (2008). Optical transmission properties and electric field distribution of interacting 2D silver nanorod Arrays. Advanced Functional Materials, 18(7)(7), 1075-1079. https://doi.org/10.1002/adfm.200701289

Published in:

Advanced Functional Materials

Queen's University Belfast - Research Portal:

Link to publication record in Queen's University Belfast Research Portal

\section{General rights}

Copyright for the publications made accessible via the Queen's University Belfast Research Portal is retained by the author(s) and / or other copyright owners and it is a condition of accessing these publications that users recognise and abide by the legal requirements associated with these rights.

Take down policy

The Research Portal is Queen's institutional repository that provides access to Queen's research output. Every effort has been made to ensure that content in the Research Portal does not infringe any person's rights, or applicable UK laws. If you discover content in the Research Portal that you believe breaches copyright or violates any law, please contact openaccess@qub.ac.uk. 


\title{
Optical Transmission Properties and Electric Field Distribution of Interacting 2D Silver Nanorod Arrays**
}

\author{
By Paul R. Evans, * René Kullock, William R. Hendren, Ron Atkinson, Robert J. Pollard, and Lukas M. Eng
}

Silver nanorods have been grown by electrodeposition into thin film porous alumina templates (AAO). Optical transmission measurements using p-polarized incident white light shows clear plasmon resonance extinction peaks. We successfully model the dependence on angle in incidence of extinction peak height and position using a multiple-multipoles (MMP) approach with the different spectral features being clearly associated with the effective electric field distribution and coupling between individual nanorods.

\section{Introduction}

Surface plasmon resonances (SPRs) excited by an incident photon beam and provoking a collective oscillation of conduction electrons have a vast number of potential applications in a diverse range of prospective devices such as optical computing, optical switching, ${ }^{[1-3]}$ biosensors ${ }^{[4]}$ and cancer treatment. ${ }^{[5]}$ Silver and gold metal nanoparticles (MNPs) have attracted particular attention due to their strong interaction in the visible and NIR regimes, while an extension of plasmon resonance into the UV light regime was demonstrated for $\mathrm{Al} \mathrm{MNPs}{ }^{[6]}$ and hole arrays. ${ }^{[7]}$ Most work on silver nanorods has been carried out with chemically grown samples produced by 'soft template' methods ${ }^{\left[{ }^{8-11]}\right.}$ that have shown excellent plasmon resonances. Other popular techniques used for forming silver nanorods and nanowires are electroplating into polycarbonate ${ }^{[12,13]}$ or alumina ${ }^{[14-21]}$ templates. The latter techniques have the advantage that nanorods or nanowires with larger aspect ratios and vertical alignment can be grown. Good Raman enhancement has also been shown by silver nanorods grown under oblique angle deposition. ${ }^{\text {[22] }}$

This paper focuses on the extension of confined SPRs to silver nanorods of $\sim 300 \mathrm{~nm}$ in length and a $22 \mathrm{~nm}$ diameter, allowing for the excitation of specific SPRs in the visible optical regime. Moreover, since the silver nanorods are arranged in a

[*] Dr. P. R. Evans, Dr. W. R. Hendren, Prof. R. Atkinson, Dr. R. J. Pollard Centre for Nanostructured Media

Queen's University Belfast

Belfast BT7 1NN (UK)

E-mail: p.r.evans@qub.ac.uk

Prof. L. M. Eng, R. Kullock

Institute of Applied Photophysics, TU Dresden

Dresden 01062 (Germany)

[**] We acknowledge financial support through the Network of Excellence "Plasmo-Nano-Devices" in the 6th EU-framework, as well as the EPSRC (UK) quasi-hexagonal 2-dimensional array with approximately $60 \mathrm{~nm}$ inter-rod spacing, near-field coupling between the nanorods becomes important. This leads to several new optical features as well as allowing the electric field distribution in such a device to be tuned. We focus here on silver (Ag) nanorod arrays owing to the particularly good scattering properties of this material in the visible optical and hence the potential for useful applications. In order to maximize these effects, it is essential to understand the electromagnetic field distribution and its spatial and temporal variation in such a device as a function of aspect ratio (=length vs. width) and spacing of the nanorods. ${ }^{[23]}$ The experimental observations presented here are accompanied by a rigorous theoretical analysis to test the validity of the modeling that may be used, ultimately, to optimize device structure for best performance

\section{Results}

\subsection{Transmission Spectra}

The nanorod arrays are produced by direct electroplating into thin film alumina (AAO) templates grown on glass substrates. ${ }^{[24]}$ Transmission spectra were measured at different angles of incidence using an Unicam UV4 UV-Vis spectrometer with the addition of a polarizer in the incident beam (Fig. 1).

Figure 2 displays the extinction spectra $\left[-\ln \left(\mathrm{I} / \mathrm{I}_{0}\right)\right]$ for a 2D-Ag nanorod carpet array still embedded in the alumina matrix measured with p-polarized white light for different incident angles. The nanorods are $300 \pm 10 \mathrm{~nm}$ long and have a diameter of $20 \pm 2 \mathrm{~nm}$ (aspect ratio of 15). The inter-rod spacing was fixed to $60( \pm 5) \mathrm{nm}$. The anodized alumina film matrix (AAO) also measures $300 \mathrm{~nm}$ in thickness, and hence the silver wires were grown to the top of the pores - as indicated by an increasing deposition current.

At normal $\left(0^{\circ}\right)$ incidence (perpendicular to the substrate) only a single broad peak is observed at $\sim 375 \mathrm{~nm}$ (black curve in 




Figure 1. Schematic diagram of the experimental set-up used for measuring optical transmission spectra of the nanorods, both with and without the silver nanorods being embedded in the thin film porous alumina matrix.

Fig. 2). By comparison with the absorbance spectra of electrochemically ${ }^{[15,20]}$ and chemically ${ }^{[8,11]}$ grown silver nanorods this peak at shorter wavelength can be assigned to the transverse resonance of the silver nanorods. As the angle of incidence is increased to $20^{\circ}$, a second peak is observed to appear centered at $\sim 572 \mathrm{~nm}$. The second peak at longer wavelength is associated with the longitudinal axis of the silver nanorods and measurements with s-polarized incident light (inset in Fig. 3) tend to support this finding since only one single absorption peak is observed, positioned at $375 \mathrm{~nm}$, for all angles of incidence. However it should be noted that the Gans theory for isolated ellipsoids ${ }^{[25]}$ would predict a longitudinal peak in the infra-red, not the visible, for silver nanorods with aspect ratio 15 . As we will show the peak we observe is actually a collective property of the nanorod array due to strong coupling between the nanorods. Note that, due to the nanorod being vertically aligned, we do not excite the electric field directly along the long axis of the nanorods. This is an important difference from nanorods in solution or dispersed horizontally on a substrate.

As the angle of incidence of the p-polarized light is increased further to $30^{\circ}$ (Fig. 2) we see that the longitudinal related peak

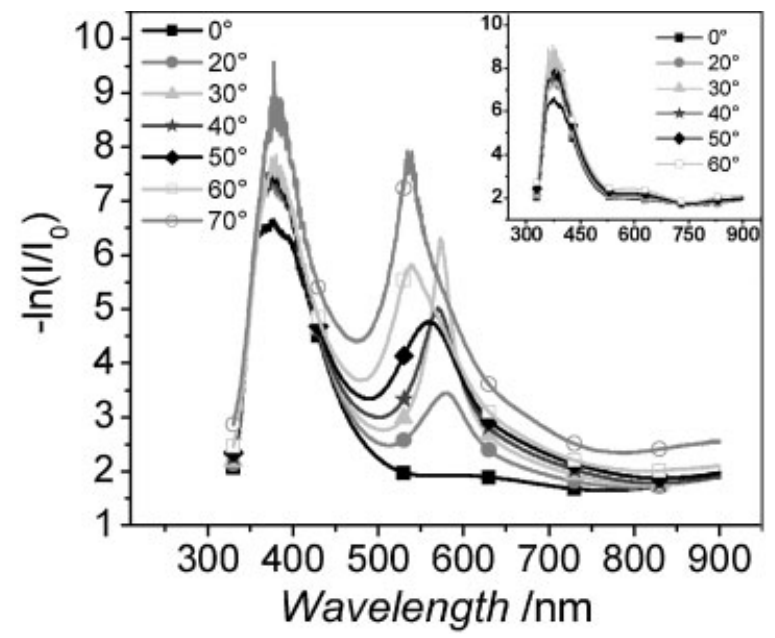

Figure 2. Extinction spectra using p-polarized incident light for various angles of incidence of $300 \mathrm{~nm}$ long silver nanorods with $20 \mathrm{~nm}$ diameter and $60 \mathrm{~nm}$ spacing embedded in a $300 \mathrm{~nm}$ thick film of porous alumina. The inset shows the same measurement for s-polarized incident light.
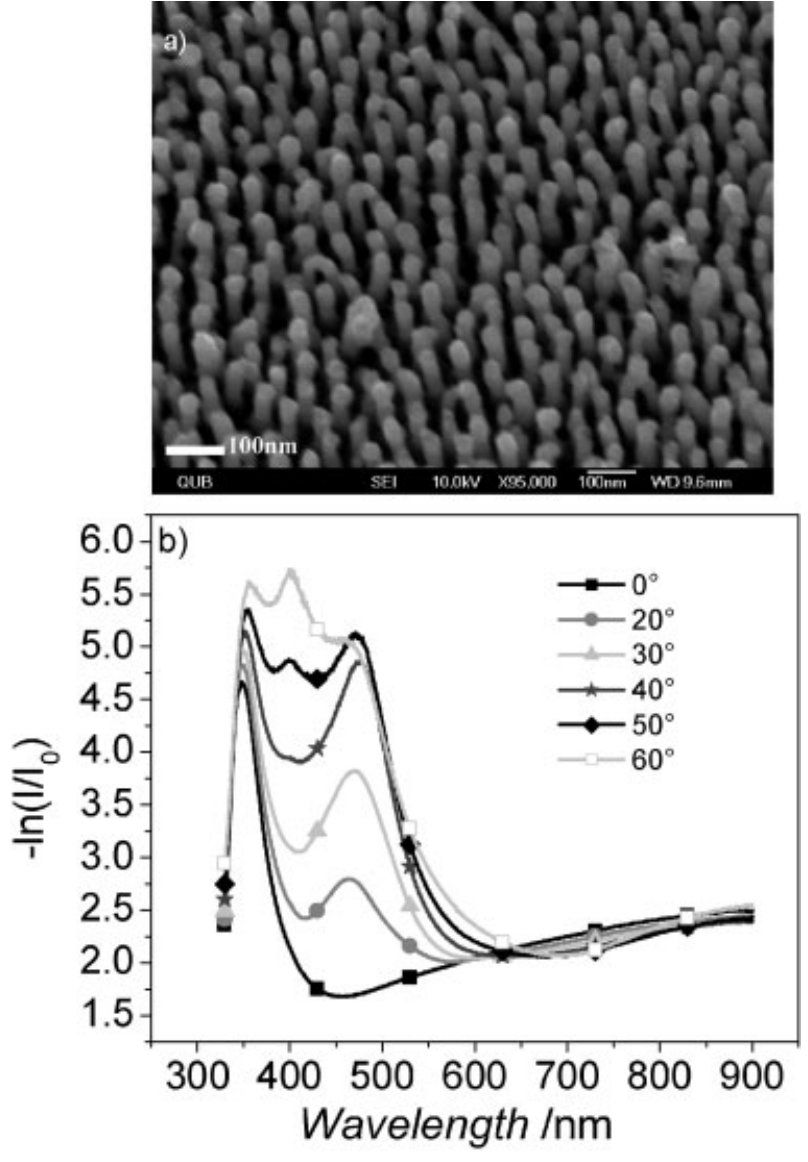

Figure 3. (a) SEM image of Silver nanorods after complete removal of the alumina matrix. (b) Extinction spectra for various angles of incidence of $300 \mathrm{~nm}$ long silver nanorods, $22 \mathrm{~nm}$ diameter, $60 \mathrm{~nm}$ spacing, after complete removal of the alumina.

increases in magnitude. However, as the angle of incidence is increased again to $40^{\circ}$, a sudden decrease in the oscillator strength and a small broadening is observed. As the angle is increased further the absorbance of the longitudinal related peak decreases before increasing again but this time at a lower wavelength of $\sim 537 \mathrm{~nm}$, a considerable peak shift of $35 \mathrm{~nm}$. Similar behavior was noted for gold nanorods without explanation in a previous paper. ${ }^{[24]}$ It should be noted that this behavior is related to nanorod length and is not seen for shorter $(<200 \mathrm{~nm}$ long) silver nanorods.

Next the alumina was removed by etching it in $\mathrm{NaOH}$ $(75 \mathrm{mM})$ to leave an array of silver nanorods in air. Figure $3 \mathrm{a}$ depicts a SEM image of the sample after complete removal of the alumina, while Figure $3 \mathrm{~b}$ shows the corresponding optical transmission data for p-polarized incident light. For zero angle of incidence it can be seen that the transverse peak of the silver nanorods has now blue shifted from $375 \mathrm{~nm}$ to $348 \mathrm{~nm}$ and become narrower compared with before etching. As the angle of incidence is further increased the peak at $470 \mathrm{~nm}$ increases $\left(<60^{\circ}\right)$ and above $40^{\circ}$ an additional peak at $\sim 400 \mathrm{~nm}$ is also observed. 


\subsection{Multiple-Multipoles Modeling}

The change in peak position and the decrease in the extinction with increasing angle of incidence of the longitudinal related peak for $300 \mathrm{~nm}$ long silver nanorods embedded in the alumina matrix (Fig. 2) is unexpected and cannot be explained using the modified Maxwell-Garnett theory we have used previously. ${ }^{[26}$ This also holds for the appearance of a 'third peak' at $\sim 400 \mathrm{~nm}$ in the extinction spectra of arrays of silver nanorods at higher angles of incidence (Fig. 3b). In order to gain a more fundamental understanding of this behavior, we modeled both cases using the semi-analytical method of multiple-multipoles (MMP). ${ }^{[27,28]}$ MMP allows for 3D calculations both in the electromagnetic near- and far-field, and hence sheds light on both aspects. Note that previously reported theoretical calculations using the finite element and Maxwell-Garnett theory focused either on the near-field ${ }^{[29,30]}$ or the far-field ${ }^{[26]}$ aspects only. MMP offers a concise and scale independent converging theoretical approach for modeling the nanorod array structures presented here.

The MMP model consists of $\mathrm{Ag}^{[31]}$ nanorods that are periodically arranged in a regular two dimensional and infinite square lattice with lattice size d (Fig. 4a). As the basis functional solutions, plane waves, different multipoles as well as the Rayleigh expansions were used as sketched in Figure $4 b$.

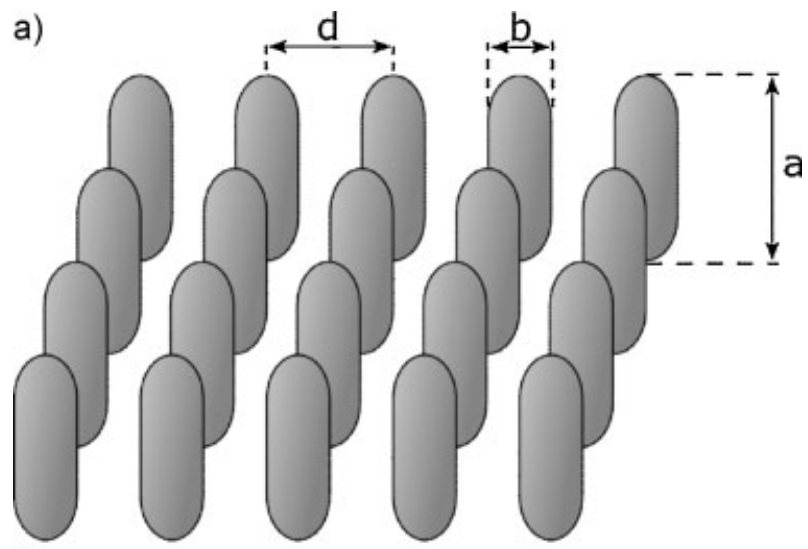

b)

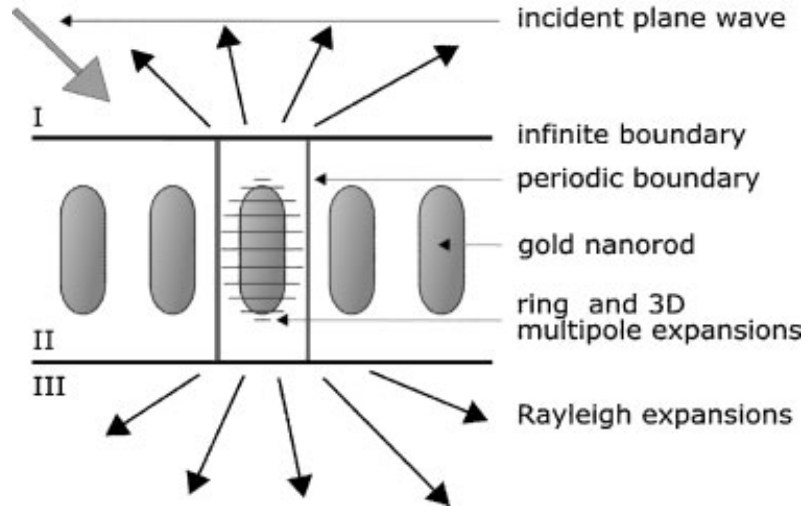

Figure 4. Schematics of the theoretical model: a) periodic arrangementb) cross section of the MMP model.
Rayleigh expansions are periodic functions describing either evanescent fields or plane waves. Their benefit is they allow an automated calculation of their orientation using periodical boundary conditions and the Floquet criterion. In order to calculate transmission spectra in accordance with the experiment, the nanorod arrangement was excited with a p-polarized incident plane wave. Then, all the necessary expansion coefficients were calculated and the amplitudes of the transmitted plane wave, which is one of the Rayleigh expansions, were recorded in the forward direction for several wavelengths and variable incident angles. For larger distances $(d>\lambda)$ and small nanorod sizes $(a, b)$ the resulting extinction spectra converged to the spectra of the quasi-electrostatic approximation of the extended Mie-Theory for a single spheroid. ${ }^{[32]}$ Such a convergence is required in order to demonstrate the validity of the model. Furthermore, for investigating the SPR modes inside the structure, the near-field distribution of the electric field was obtained using the calculated expansion coefficients of the multipoles.

Figures 5 and 6 illustrate the theoretically obtained extinction spectra for the silver nanorod arrays, under p-illumination, with and without the AAO matrix respectively. For the silver nanorods still embedded in the AAO matrix (see Figs. 2 and 5) the calculated extinction spectra show the same basic behavior as was observed for the experimental data:

(1) a single peak at $\sim 380 \mathrm{~nm}$ for normal incidence $\left(0^{\circ}\right)$ which remains stationary for increasing angle

(2) A peak at long wavelength that initially increases with the angle of incidence and (3) then decreases as a second corresponding peak at slightly shorter wavelength increases.

The magnitudes of the extinction peaks, as well as the position of the peak at $380 \mathrm{~nm}$, all show good agreement with

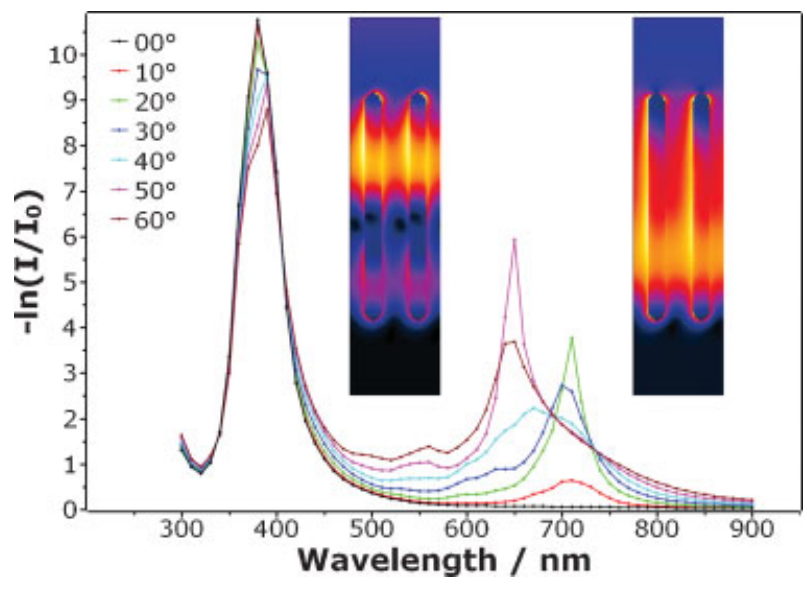

Figure 5. Calculated optical extinction spectra using p-polarized light for various angles of incidence. The array consists of individual $\mathrm{Ag}$ nanorod measuring $300 \mathrm{~nm}$ and $22 \mathrm{~nm}$ in length and diameter respectively, and a $60 \mathrm{~nm}$ spacing between the rods. Note that these nanorods are fully embedded in a AAO matrix with $n=1.6$. Insets: Field plots of the averaged electromagnetic field at the two resonances $\left(20^{\circ}, 710 \mathrm{~nm}\right)$ and $\left(50^{\circ}\right.$, $650 \mathrm{~nm}$ ). Colour range: yellow - high field strength; black - low field strength. To emphasise the mode structure the plots are not to scale. 


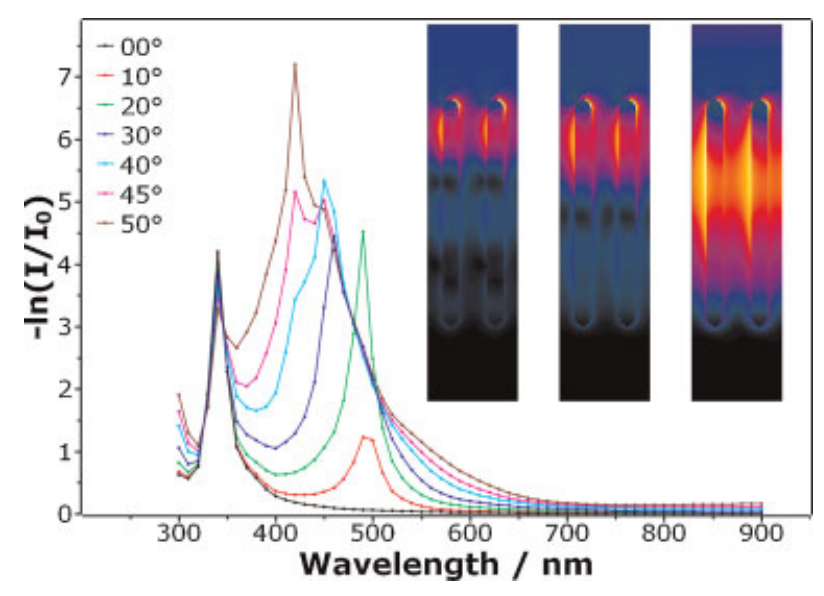

Figure 6. Calculated extinction spectra using p-polarized light for various angles of incidence. The array consists of individual $\mathrm{Ag}$ nanorod measuring $300 \mathrm{~nm}$ and $22 \mathrm{~nm}$ in length and diameter, respectively, and a $60 \mathrm{~nm}$ spacing between the rods. Note that these nanorods are embedded in an environment with $n=1.0$ (air). Insets: Field plots of the averaged electromagnetic field at the resonances $\left(50^{\circ}, 420 \mathrm{~nm}\right),\left(40^{\circ}, 450 \mathrm{~nm}\right)$ and $\left(20^{\circ}, 490 \mathrm{~nm}\right)$. Colour range: yellow - high field strength; black low field strength. To emphasise the mode structure the plots are not to scale.

experiment. However, the positions of the two 'longitudinal' related extinction peaks are predicted to be $\sim 100 \mathrm{~nm}$ redshifted from where they are actually observed $(710 \mathrm{~nm}$ vs. $570 \mathrm{~nm}$, and $650 \mathrm{~nm}$ vs. $535 \mathrm{~nm}$ ). Also the maximum extinction with angle for the peak at the longest wavelength is predicted to occur at an angle of incidence of $20^{\circ}$, whilst experimentally it is $30^{\circ}$. The reasons for this discrepancy will be dealt with in the next section. It is also interesting to note in Figure 5 that the 'transverse' extinction peak at $380 \mathrm{~nm}$ is predicted to decrease in height with increasing angle. Whilst this behavior is not seen for the $300 \mathrm{~nm}$ long silver nanorods it is observed for shorter $(150 \mathrm{~nm}-200 \mathrm{~nm})$ silver rods embedded in alumina. This may indicate that multipole plasmon resonances within a nanorod $^{[33]}$ play a role but additional evidence is needed.

For the bare silver nanorod arrays (AAO matrix removed Fig. 6) the normal incidence extinction peak is predict to red shift to $340 \mathrm{~nm}$, which is in good agreement with what is experimentally observed (Fig. 4b). As the angle of incidence increases, peaks are predicted at $490 \mathrm{~nm}\left(20^{\circ}\right), 450 \mathrm{~nm}\left(40^{\circ}\right)$ and $420 \mathrm{~nm}\left(50^{\circ}\right)$. Experimentally (Fig. 3b) a peak which increases with angle at $470 \mathrm{~nm}$ and a second peak that appears above $50^{\circ}$ at $400 \mathrm{~nm}$ are observed.

\section{Discussion}

The theoretical calculations can also provide insight into the electric field distribution and coupling between plasmons bound to the nanorods. The insets in Figures 5 and 6 illustrate these electromagnetic field plots associated with the different extinction peaks. Each plot shows a cross section through two neighbouring nanorods (out of the infinite arrangement). The electric field is averaged over a whole temporal period of the incident light. For both cases - with or without AAO - the peaks with the lowest energy are correlated to the $1^{\text {st }}$-order mode having the field maximum in the middle of the structure, i.e. approximately at half-length. The field plot of the next peak shows a node in the middle of the structure and two maxima below and above that node. It is therefore associated with the $2^{\text {nd }}$-order mode. In the case without AAO (Fig. 6) it is even possible to see the $3^{\text {rd }}$-order mode which has two nodes, three maxima and thus is associated with the peak having the third lowest energy. The peak with the highest energy is always correlated to the short axis or $0^{\text {th }}$-order mode. Note, the field strength decreases gradually from top to bottom due to plasmon damping leading to smaller anti-nodes at the bottom than at the top for the $2^{\text {nd }}$ - and $3^{\text {rd }}$-order modes.

Nevertheless, it is possible to clearly distinguish the different plasmon modes from each other. Although they look similar to the modes of single metal nanorods as shown by ${ }^{[33-35]}$ they differ in several important aspects owing to the nanorod arrangement. Firstly, because the nanorods are closely spaced there is a strong coupling between the plasmons of neighboring nanorods, ${ }^{[26,30]}$ which leads to a peak being observed which, whilst related to the longitudinal resonance for an isolated nanorod, is actually a collective property of the nanorod array and is significantly blue shifted from what would be predicted for an isolated nanorod. ${ }^{[36]}$. And secondly, because the nanorods are vertically aligned, the different modes are only excitable for certain angles of incidence. For noninteracting silver or gold nanorods, for example in solution, the 'hot spot' of maximum field intensity is normally located at the end of the rod. ${ }^{[7]}$ However the calculations for our structure show that the interaction between the gold and silver nanorods leads to the 'hot spot' being located between the nanorods. This has some advantage for fluorescence where the close proximity of metal particles can lead to quenching.

The red-shift and the smaller resonance angles of the calculated results for silver nanorods embedded in the alumina matrix compared to the measured spectra may be attributed to the following issues:

(1) As the actual dielectric function of these Ag nanorods is not directly accessible, we used bulk values for silver ${ }^{[31]}$ as reported in literature. However, depending on the choice of bulk data-sets, the centre peak positions were found to vary considerably and could scatter over a $40 \mathrm{~nm}$ spectral range.

(2) To simplify the model, the $5 \mathrm{~nm}$ gold and $20 \mathrm{~nm} \mathrm{Ta}_{2} \mathrm{O}_{5}$ base electrode layer was neglected in the calculation.

(3) The nanorod geometry used for the calculation was quadratic (Fig. 2a), whilst ordered alumina is usually quasi-hexagonal. ${ }^{[37]}$ This may lead to a different coupling strength between the nanorods.

(4) Imperfections of the samples - both in terms of periodicity and structure of the electroplated silver — may lead to a broadening of spectral features and hence to a significant peak overlap. In fact, this is presumably the case for the structure measured without any AAO matrix where the first two peaks of the simulated spectrum may be appearing as only a single experimental peak. 


\section{Conclusions}

We have fabricated 2D silver nanorod arrays by electrochemical growth into thin film porous alumina templates and measured their extinction spectra in the visible regime. An unusual dependence of the extinction peak height and position on angle of incidence was observed for silver nanorod arrays embedded in alumina. Three extinction peaks were observed at high angles of incidence for bare silver nanorod arrays. A multiple multipole approach has been used to model the optical behavior of the arrays for incident p-polarized light and it has been found that these various resonances can clearly be associated with plasmon modes of different orders.

The nanorod array arrangement allows the optical transmission to be tuned into a spectral regime with optimal characteristics, i.e. either selecting high absorptive or transmissive properties. In addition our calculations allow us to make predictions for any material applicable to the spectral range of interest, i.e. producing nanorod arrays with exactly known optical features.

\section{Experimental}

The nanorod arrays are produced by direct electroplating into thin film alumina (AAO) templates grown on glass substrates [24]. In order to prepare the alumina templates, first a $20 \mathrm{~nm}$ thick layer of tantalum oxide was deposited onto a glass substrate by reactive ion sputtering. A $5 \mathrm{~nm}$ gold layer followed by a $300-450 \mathrm{~nm}$ thick aluminum was then sputtered onto the tantalum oxide. The aluminum was subsequently anodized in $0.3 \mathrm{M}$ sulfuric acid at a typical voltage of $30 \mathrm{~V}$ to produce pores with a $20 \pm 4 \mathrm{~nm}$ diameter and $50 \pm 6 \mathrm{~nm}$ spacing. A platinum counter electrode was used for the anodization and the electrolyte was kept at a constant temperature of $2{ }^{\circ} \mathrm{C}$. Prior to electrodeposition the templates were etched in $30 \mathrm{mM}$ sodium hydroxide for 35 seconds in order to remove the barrier layer at the bottom of each pore. The etching time determines the pore diameter and hence the nanorod cross-section. Silver was electrodeposited under potentiostatic control at a voltage of $-0.3 \mathrm{~V}$ vs. a saturated calomel electrode (SCE). The electrolyte used consisted of $\mathrm{AgBr}(0.1 \mathrm{M})$, sodium sulfite $(0.2 \mathrm{M})$ and sodium thiosulfate $(0.25 \mathrm{M})$ in water [38].

Transmission spectra were measured at different angles of incidence using an Unicam UV4 UV-Vis spectrometer with the addition of a polarizer in the incident beam (Fig. 1). The sample structure was characterized using a Jeol $6500 \mathrm{~F}$ field emission scanning electron microscope (SEM)

Received: November 4, 2007 Revised: January 14, 2008 Published online: March 31, 2008

[1] G. A. Wurtz, R. J. Pollard, A. V. Zayats, Phys. Rev. Lett. 2006, 97, 057402.

[2] S. W. Liu, M. Xao, Appl. Phys. Lett. 2006, 88, 143512.

[3] T. Nikolajsen, K. Leosson, S. I. Bozhevolyni, Appl. Phys. Lett. 2004, $85,5833$.

[4] G. Raschke, S. Kowarik, T. Franzl, C. Sonnichsen, T. A. Klar, J. Feldmann, A. Nichtl, K. Kulrzinger, Nano Lett. 2003, 3, 935.
[5] X. Huang, I. H. El-Sayed, W. Qian, M. A. El-Sayed, J. Am. Chem. Soc. 2006, 128, 2115.

[6] Y. Ekinci, H. H. Solak, J. F. Löffler, Adv. Funct. Mater. in press.

[7] Y. Ekinci, H. H. Solak, C. David, Opt. Lett. 2007, 32, 172.

[8] Y. Sun, Y. Yadong, B. T. Mayers, T. Herricks, Y. Xia, Chem. Mater. 2002, 14, 4736.

[9] Y. Sun, Y. Yadong, B. T. Mayers, T. Herricks, Y. Xia, Nano Lett. 2003, 3, 955.

[10] N. R. Jana, L. Gearheart, C. J. Murphy, Chem. Commun. 2001, 7, 617

[11] C. J. Murphy, T. K. Sau, A. M. Gole, C. J. Orendorff, J. Gao, L. Gou, S. E. Hunyadi, T. Li, J. Phys. Chem. B. 2005, 109, 13857.

[12] J. J. Mock, S. J. Oldenburg, D. R. Smith, D. A. Schultz, S. Schultz, Nano Lett. 2002, 2, 465.

[13] I. Kazeminezhad, A. C. Barnes, J. D. Holbrey, K. R. Seddon, W. Schwarzacher, App. Phys. A Mater. Sci. Processing 2007, 86, 373.

[14] R.-L. Zong, J. Zhou, Q. Li, L. Li, W. Wang, Z. Chen, Chem. Phys. Lett. 2004, 398, 224.

[15] R.-L. Zong, J. Zhou, Q. Li, B. Du, B. Li, M. Fu, X.-W. Qi, L.-T. Li, S. Buddhudu, J. Phys. Chem. B 2004, 108, 16713.

[16] J. Choi, G. Sauer, K. Nielsch, R. B. Wehrspohn, U. Gösele, Chem. Mater. 2003, 15, 776.

[17] G. Sauer, G. Brehm, S. Schneide, K. Nielsch, R. B. Wehrspohn, J. Choi, H. Hofmeister, U. Gösele, J. App. Phys. 2002, 91, 3243.

[18] T. R. Kline, M. Tian, J. Wang, A. Sen, M. W. H. Chan, T. E. Mallouk, Inorg. Chem. 2006, 45, 7555 .

[19] B. L. Broglin, A. Andreu, N. Dhussa, J. A. Heath, J. Gerst, B. Dudley, D. Holland, M. El-Kouedi, Langmuir 2007, 23, 4563.

[20] G. Sauer, G. Brehm, S. Schneide, H. Graner, G. Seifert, K. Nielsch, J Choi, P. Göring, U. Gösele, P. Miclea, R. B. Wehrspohn, J. App. Phy. 2005, 97, 024308

[21] G. H. Gu, J. Kim, L. Kim, J. S. Suh, J. Phys. Chem. C 2007, 111, 7906.

[22] S. B. Chaney, S. Shanmukh, R. A. Dluhy, Y.-P. Zhao, Appl. Phys. Lett. 2005, 87, 031908.

[23] H. E. Ruda, A. Shik, App. Phy. Lett. 2007, 90, 223106.

[24] P. Evans, W. R. Hendren, R. Atkinson, G. A. Wurtz, W. Dickson, A V. Zayats, R. J. Pollard, Nanotechnology 2006, 17, 5746.

[25] R. Gans, Ann. Phys. 1915, 4, 270.

[26] R. Atkinson, W. Hendren, G. Wurtz, W. Dickson, A. V. Zayats, P. Evans, R. Pollard, Phys. Rev. B 2006, 73, 235402.

[27] C. Hafner, MaX-1 A Visual Electromagnetics Platform for PCs, John Wiley \& Sons, Chichester, UK 1998.

[28] C. Hafner, Post-modern Electromagnetics: Using Intelligent MaXwell Solvers, John Wiley \& Sons, Chichester, UK 1999.

[29] P. R. Evans, G. A. Wurtz, R. Atkinson, W. R. Hendren, W. Dickson, R. J. Pollard, A. V. Zayats, J. Phys. Chem. C 2007, 111, 12522.

[30] W. Dickson, G. A. Wurtz, P. Evans, D. O'Connor, R. Atkinson, R. J. Pollard, A. V. Zayats, Phys. Rev. B 2007, 76, 115411.

[31] D. W. Lynch, W. R. Hunter, Handbook of Optical Constants of Solids II, 2nd ed., Academic Press, New York 1985, p. 350.

[32] C. F. Bohren, D. R. Huffman, Absorption and Scattering of Light by Small Particles, John Wiley \& Sons, New York 1998.

[33] E. Payne, K. Shuford, S. Park, G. Schatz, C. Mirkin, J. Phys. Chem. B 2006, 110, 2150.

[34] K. Imura, T. Nagahara, H. Okamoto, J. Chem. Phys. 2005, 122, 154701.

[35] B. N. Khlebtsov, N. G. Khlebtsov, J. Phys. Chem. C 2007, 111, 11516.

[36] L. Novotny, Phys. Rev. Lett. 2007, 98, 266802.

[37] H. Masuda, K. Fukada, Science 1995, 268, 1466.

[38] D. G. Foster, Y. Shapir, J. Jorne, J. Electrochem. Soc. 2003, 150, C375. 